and take

$$
X_{0}=\frac{1}{2} A^{*}=\frac{1}{2}\left(\begin{array}{cc}
1 & 0 \\
0 & 1 \\
-1 & 1
\end{array}\right)
$$

Here, formula (17) is used to obtain:

$$
\begin{aligned}
X_{1} & =\frac{1}{2}\left(\begin{array}{cc}
1 & 0 \\
0 & 1 \\
-1 & 1
\end{array}\right)\left\{2\left(\begin{array}{ll}
1 & 0 \\
0 & 1
\end{array}\right)-\frac{1}{2}\left(\begin{array}{ccc}
1 & 0 & -1 \\
0 & 1 & 1
\end{array}\right)\left(\begin{array}{cc}
1 & 0 \\
0 & 1 \\
-1 & 1
\end{array}\right)\right\} \\
& =\frac{1}{4}\left(\begin{array}{cc}
2 & 1 \\
1 & 2 \\
-1 & 1
\end{array}\right), \\
X_{2} & =\frac{1}{16}\left(\begin{array}{cc}
10 & 5 \\
5 & 10 \\
-5 & 5
\end{array}\right), \\
X_{3} & =\frac{1}{256}\left(\begin{array}{cc}
170 & 85 \\
85 & 170 \\
-85 & 85
\end{array}\right), \quad \text { etc. }
\end{aligned}
$$

converging to:

$$
A^{+}=\frac{1}{3}\left(\begin{array}{cc}
2 & 1 \\
1 & 2 \\
-1 & 1
\end{array}\right) .
$$

Technion, Israel Institute of Technology

Haifa, Israel

1. G. Schulz, "Iterative Berechnung der reziproken Matrix," Z. Angew. Math. Mech. v. 13,1933 , pp. $57-59$.

2. R. Penrose, "A generalized inverse for matrices," Proc. Cambridge Philos. Soc., v. 51, 1955, pp. 406-413. MR 16, 1082.

3. A. Ben-IsRael \& A. Charnes, "Contributions to the theory of generalized inverses," J. Soc. Indust. Appl. Math., v. 11, 1963, pp. 667-699.

4. W. Dück, "Fehlerabschätzungen für das Iterationsverfahren von Schulz zur Bestimmung der Inversen einer Matrix," $Z$. Angew. Math. Mech., v. 40, 1960, pp. 192-194. MR 22 \#3102. 1964 .

5. A. S. Housenolder, Theory of Matrices in Numerical Analysis, Blaisdell, New York,

\title{
A Note on the Maximum Value of Determinants over the Complex Field
}

\author{
By C. H. Yang
}

The purpose of this note is to extend a theorem on determinants over the real field to the corresponding theorem over the complex field.

Theorem. Let $D(n)$ be an nth order determinant with complex numbers as its entries. Then

$$
\operatorname{Max}_{\left|a_{j k}\right| \leqq K}|D(n)|=\operatorname{Max}_{\left|a_{j k}\right|=K}|D(n)| .
$$

Received June 5, 1964. Revised December 8, 1964. 
In other words, $D(n)$ is a function of $n^{2}$ variables $a_{j k}$ which vary over the bounded and closed domain $\bar{D}:\left\{\left|a_{, k}\right| \leqq K\right\}$; hence this function is bounded and attains its maximum value on the boundary of the domain $\bar{D}$.

Proof. Let $a_{j k}=r_{j k} e^{i i_{j k}}$ and $A_{j k}=R_{j k} e^{i \phi_{j k}}=$ the co-factor of $a_{j k}$, where $K \geqq r_{j k} \geqq 0$ and $R_{j k} \geqq 0$. Then, expanding by the $j$ th row, we have

$$
\begin{aligned}
|D(n)| & =\left|\sum_{k=1}^{n} a_{j k} A_{j k}\right|=\left|\sum_{k=1}^{n} r_{j k} R_{j k} e^{i\left(\boldsymbol{o}_{j k}+\phi_{j k}\right)}\right| \\
& \leqq \sum_{k=1}^{n} r_{j k} R_{j k} \leqq \sum_{k=1}^{n} K R_{j k}=D^{\prime}(n),
\end{aligned}
$$

where $D^{\prime}(n)$ is the $n$th order determinant whose entries are

$$
a_{j k}^{\prime}= \begin{cases}a_{j k}, & \text { if } r_{j k}=K \text { and } \theta_{j k}+\phi_{j k} \equiv 0(\bmod 2 \pi) \\ K e^{-i \phi j k}, & \text { if } r_{j k}<K \text { or } \theta_{j k}+\phi_{j k} \neq \equiv(\bmod 2 \pi) .\end{cases}
$$

By applying the same process to the other rows, we obtain a determinant $D^{*}(n)$ whose entries $\left|a_{j k}^{*}\right|=K$ and $\left|D^{*}(n)\right| \geqq|D(n)|$. Hence, $\operatorname{Max}_{\left|a_{j k}\right| \leq K}|D(n)| \leqq$ $\operatorname{Max}_{\left|a_{j k}\right|-x}|D(n)|$; thus the proof of the theorem can be completed since the reverse inequality is trivial.

50 Mohigan Drive

Oneonta, New York

\section{On the Numerical Solution of $y^{\prime}=f(x, y)$ by a Class of Formulae Based on Rational Approximation}

\section{By John D. Lambert and Brian Shaw}

1. Introduction. Most finite difference formulae in common usage for the numerical solution of first-order differential equations are based on polynomial approximation. Two exceptions are the formulae based on exponential approximation proposed by Brock and Murray [1], and the formulae of Gautschi [2] which are derived from trigonometric polynomials. The use of rational functions as approximants has been studied by many authors, including Remes [3], Maehly [4] and Stoer [5], but the main concern of most of this work has been the direct approximation of a given function. Algorithms for interpolation based on rational functions have been proposed by Wynn [6], and methods for numerical integration and differentiation based on Padé approximation have been studied by Kopal [7]. It is the purpose of the present paper to derive a class of formulae, based on rational approximation, for the numerical solution of the initial value problem

$$
y^{\prime}=f(x, y), \quad y\left(x_{0}\right)=y_{0} .
$$

The formulae proposed give exact results when the theoretical solution of (1) is a rational function of a certain degree, just as many of the classical difference formulae

Received December 10, 1964. 